\title{
Engineering Biocompatible Hydrogel Fibers With Tunable Mechanical Properties For Neural Tissue Engineering
}

\section{Roya Samanipour}

University of British Columbia

Hamed Tahmooressi

University of British Columbia

Hojatollah Rezaei Nejad

Tufts University

\section{Kabilan Sakthivel}

University of British Columbia

\section{Adel Yavarinasab}

University of British Columbia

\section{Bahram Talebjedi}

University of British Columbia

Nishat Tasnim

University of British Columbia

Mina Hoorfar ( $\nabla$ mina.hoorfar@ubc.ca )

University of British Columbia

\section{Research Article}

Keywords: neural tissue, hydrogel scaffold, bioprinting, microfluidics, cell culture

Posted Date: March 1st, 2021

DOI: https://doi.org/10.21203/rs.3.rs-240546/v1

License: (c) (1) This work is licensed under a Creative Commons Attribution 4.0 International License. Read Full License 


\section{Abstract}

Neural tissue engineering holds a great promise for the treatment of neurodegenerative diseases and peripheral nerve injuries. However, the anisotropic mechanical and electrical properties of the highly aligned neural cells have hindered the development of a faithful in vitro disease models. In this study, a core-shell microfluidic extrusion method is implemented to fabricate a cell-laden composite hydrogel fiber with tunable mechanical properties. The hybrid hydrogel was formed using a core of GelMA mixed with gelatin seeded with human neuroblastoma cell (SH-SY5Y) and an alginate shell. The composition of the core hydrogel was optimized to support cellular growth and differentiation, yet allow a feasible fabrication of cell-laden fibers. The engineered fibers were remarkably biocompatible and enabled the formation of highly aligned cellular morphology.

\section{Introduction}

The central nervous system (CNS) has a limited capacity to grow new cells to repair the loss or damage of neurons and axonal pathways that accompany conditions such as traumatic brain injury (TBI), stroke, spinal cord injury, and neurodegenerative diseases ${ }^{1-4}$. Neurological disorders (such as stroke, dementia, meningitis, etc.) are among the leading causes of disability ( $~ 276$ million) and deaths ( 9 million in 2016) which continue to increase each year ${ }^{5}$. Neurogenesis is a multi-step process of neural stem cell differentiation in the CNS; however, it is restricted to a limited number of areas in the brain (e.g. hippocampus dentate gyrus, etc.), hampering the restoration of the lost neurons ${ }^{6,7}$. Additionally, regeneration of lost axonal pathways in the CNS is insufficient due to the lack of directed guidance as well as inhibitors like glial scar or myelin ${ }^{8,9}$. Conventional regenerative strategies to treat neurological diseases, including gene therapy, stem cell transplantation/engraftment or growth factor delivery, have shown limited success in creating functional tissues due to the low cell engraftment and lack of growth factors localization ${ }^{10,11}$. Animal model, as a traditional alternative, is extensively employed for the regenerative medicine experiments and CNS disorder therapies ${ }^{12,13}$. However, the differences in animal models compared to human body, in addition to the high costs and related ethical issues, have posed serious concerns on the applicability of animal models ${ }^{14}$. Regarding these critical issues, numerous measures have been implemented to develop reliable in vitro disease models ${ }^{15-17}$.

Different in vitro techniques such as soft lithography ${ }^{18}$, laser texturing ${ }^{19}$, electrospinning ${ }^{20}$, and electrospraying ${ }^{21}$, have been employed to generate 2D cell-laden constructs. However, these techniques fail to accurately mimic the mechanical properties, architecture and physiology of brain-specific structure and function ${ }^{22}$. In fact, in 2D culture methods, the functionality of neural cells (including morphology, proliferation, and differentiation) is either limited or completely lost ${ }^{23,24}$. In contrast to the $2 \mathrm{D}$ methods, three-dimensional (3D) bioprinted structures have demonstrated new prospective in mimicking the functional native-like tissue microenvironment ${ }^{25,26}$. In $3 \mathrm{D}$ bioprinting, cells, biomaterial, and bioactive moieties can be precisely positioned to mimic in vivo conditions ${ }^{27,28}$. This holds a great promise for 
future advancements toward having a cost-effective, fast and anatomically accurate representation to address the current issues of CNS disorder therapies ${ }^{29-31}$.

Generally, 3D bioprinting techniques can be divided into optical-based and nozzle-based methods ${ }^{32}$. In optical-based methods, the major step is the scaffold photopolymerization where a high energy radiation is exposed to the cell-containing precursors ${ }^{33}$. This can significantly reduce the cell viability rate, as compared to nozzle-based methods ${ }^{34}$. Although the optical-based methods have higher printing resolution, the simplicity and the low cost of nozzle-based methods have made the them more prevalent. Amongst the nozzle-based bioprinting approaches, extrusion bioprinters have the advantage of generating low shear stress on living cells, making them more cytocompatible ${ }^{34}$. Another issue in mimicking neural tissues is the complex microenvironment where neurons, glial cells, blood vessels, pericytes, and extracellular matrix are elaborately bundled ${ }^{35}$. This requires manipulation of different cell types and printing materials simultaneously ${ }^{36}$. For that reason, coaxial extrusion methods can crosslink different biocompatible hydrogels (e.g. core-shell) at the same time through printing a fiber-like construct 37,38. In fact, there are two major superiorities of coaxial extrusion printing over other methods to be used for neural tissue disease models: (i) it provides guidelines for a controlled cell proliferation and axon outgrowth (fiber structure); and (ii) it can delicately position more than one cell type in a 3D tubular structure (core-shell structure).

Regarding the cell proliferation guidelines, a common approach is to use aligned polymer nanofibers to fabricate a 3D scaffold as a culture microenvironment ${ }^{39,40}$. The electrospun nanofibers can provide a controlled neurite outgrowth and cell alignment replicating the native neuroanatomical architecture ${ }^{41}$. As an example, a 3D scaffold based on a bacterial nanocellulose (BNC) can prove a biocompatible environment for neural cells (neuroblastoma, SH-SY5Y) ${ }^{42}$. The nanofibers can also mix with carbon nanotubes to create a conductive 3D composite scaffold in which an exceptional cell (SH-SY5Y) attachment and proliferation along the guidelines can occur ${ }^{43}$. In addition to nanofibers, other strategies such as chemical surface treatment, mechanical stimulation, and surface patterning can be implemented for a directional cell spreading and growth ${ }^{44}$. Although these methods can provide a relatively acceptable cell guiding platform, the complexity engaged in simultaneously manipulating of different cell types as well as the higher fabrication costs are still their ongoing challenges.

The fiber constructs when fabricated in a core-shell form provides even a broader course of in vitro applications. The structure of coaxial constructs can robustly recapitulate the luminal/tubular configuration of cell bundles of neurovascular/neuromuscular tissues in a 3D environment, rather than a planner organ-on-chip ${ }^{35,45}$. In core-shell constructs, cells located in the core/shell are protected by the outer hydrogel shell that provides both stability and the possibility of cell-cell interactions within the environment ${ }^{46}$. A longitudinal fiber-based scaffold fabricated to investigate the drug resistance in Glioblastoma, with human glioma stem cell (GSC23) in the shell and glioma cell line (U118) in the core, showed the superiority of core-shell constructs to mimic the native microenvironment of the neural tissues ${ }^{47}$. A recent study with neural stem cells (core) and Schwann cells (shell) showed an 
enhancement in cell proliferation and differentiation that can be implemented for spinal cord injury research ${ }^{48}$. In neurodegenerative disease models, however, longitudinal scaffolds do not necessarily provide a native-like microenvironment where neural cells are highly paralleled with the aligned Schwann cells ${ }^{44,49}$. In other words, growing highly aligned neural cells even in the core-shell fibers is still a challenge. Therefore, a neural tissue model should be able to align cells in an parallel orientation where the synaptic electrophysiology, plasticity, and neural vesicle trafficking can occur ${ }^{44}$.

Specifically under the topic of scaffold activated directional neural growth, only a few studies are conducted. As an instant, rat cortical cells and mouse neural stem cell were encapsulated in an ECM (extracellular matrix) protein hydrogel using the coaxial printing method. Despite the extensive investigation on different cell types in this study, the neural outgrowth and directional neural outgrowth is not quantitively measured ${ }^{50}$. Glia cells together with primary cortical neurons are also printed using a coaxial printing strategy that utilizes Gellan gum-RGD as the bioink ${ }^{37}$. Rather than the directed neurite outgrowth of the cells, networking of these types of cells was investigated in that study. Human derived cortical neurons and glial cells (derived from Human-Induced Pluripotent Stem Cells) were used in another bioprinting study ${ }^{51}$. Likewise, the cellular alignment was not the focus in that study.

In addition to CNS related diseases, peripheral nerve injuries and regeneration of neural fibers are other important applications of coaxial bioprinting ${ }^{52}$. In fact, the importance of growing neural cells along the desired direction can be realized in the regeneration of the peripheral nervous system ${ }^{53}$. In most of the studies on peripheral neural on-chip models, fabrication of a guiding conduit for directed axonal growth is of interest.

As mentioned above, in vitro cell alignment in neural tissue engineering plays a critical role to faithfully model the neurodegenerative diseases. Herein, we fabricated a core-shell hydrogel scaffold with a superior neural cell alignment within the construct. The hybrid scaffold microenvironment is comprised of gelatin methacryloyl (GelMA)/gelatin and hydrogel as the core, and an alginate as the shell. Human neuroblastoma cells (SH-SY5Y) are cultured in the fiber core with a high cell proliferation and more than $85 \%$ cell viability. Cell alignment is achieved with a standard deviation of less than 6 in cellular orientation. The microcylindrical 3D scaffold structure is shown to recapitulate the structure of the native extracellular matrix (ECM), as the neural growth is highly aligned with the fiber guidelines.

\section{Results}

\subsection{Fabrication of hybrid hydrogel fiber}

The schematic of the microfluidic setup used to fabricate the hydrogel fibers is presented in Fig. 1(A). The system is consisted of two syringe pumps, a co-flowing microfluidic chip with two inlets and one outlet, and a calcium chloride $\left(\mathrm{CaCl}_{2}\right)$ bath. The microfluidic system is made by two concentric metallic needles together creating a core and sheath stream of different fluids at the outlet. The outlet of the microfluidic 


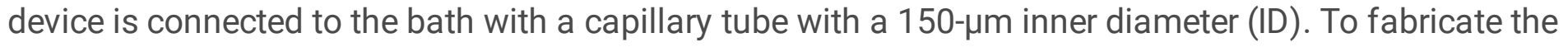
composite cell-laden hydrogel fibers, a solution of sodium alginate (shell) and a solution of GelMA/gelatin containing the neuroblastoma cells (core) are pumped through the microfluidic device (see Material and Method Section for the details) (Fig. 1(B)). The GelMA/gelatin is pumped to the core channel and the alginate is pumped to the shell channel. Then the fabricated fibers are introduced to $\mathrm{CaCl}_{2}$ bath (see Fig. 2(A)). The fiber core is made of GelMA as it provides binding sites for the neural cells ${ }^{54}$. However, wet spinning of GelMA is challenging ${ }^{55}$. Therefore, an alginate shell is formed as a support to physically entrap GelMA monomers in the fiber prior to their photocrosslinking ${ }^{56}$. Once the solutions extruded into the $\mathrm{CaCl}_{2}$ bath, calcium ions replace the sodium and create the calcium alginate hydrogel

57. The collected fibers are exposed to UV light to photocrosslink the GelMA hydrogel (Fig. 1(B)). The diameter of the core and the thickness of the shell could be tuned simply by controlling the ratio of the flow rates.

\subsection{Physical property tuning}

The setup used for the experiment is shown in Fig. 2(A). The core-shell construct in Fig. 2(B) is fabricated by $3 \% / 2 \% \mathrm{w} / \mathrm{v}$ of GelMA/gelatin ink for the core and $2 \% \mathrm{w} / \mathrm{v}$ alginate ink for the shell. The core-shell regions remained unmixed (Fig. 2(B)). The flow rates of different streams were changed to alter the dimensions of the fabricated fibers. Increasing the flow rate ratio between the inner and the outer stream increased the diameter of the printed hydrogel fiber (Fig. 2(C)). The mechanical properties of the fabricated fibers can be tailored by changing the properties of the used pre-polymers. Mechanical properties of the fibers can also be tailored by changing the alginate concentration. The results show that a higher alginate concentration yields larger Young's modulus (Fig. 2(D)). The Young's modulus of the core hydrogel material consisted of GelMA/alginate were measured for different concentrations of GelMA at $1 \%$ gelatin. Increasing the concentration of GelMA, the Young's modulus is increased (Fig. 2(E)). The Young's modulus of the $2.5 \%$ GelMA and $1 \%$ gelatin solution is about $0.6 \mathrm{kPa}$, which is around the stiffness of the native brain tissue ${ }^{58}$. Therefore, this concentration is selected as the optimized scaffold for the cell culture microenvironment. Figure 2(F) shows the Young's modulus for different concentrations of GelMA combined with $1 \%$ alginate which is higher than the stiffness of the native brain tissue.

\subsection{Cytotoxicity evaluation}

The ability of the fabrication method and the scaffold materials to recapitulate the native microenvironment for cell growth and proliferation is evaluated using different tests for the SH-SY5Y cells (Fig. 3). The lateral shell flow in the core-shell structure confines the cells to flow within the core region. Figure 3(A) shows the phase contrast images of the cells encapsulated in hydrogel fiber at Day 0,1 , and 5 of the fabrication. The viability assessment was performed considering the live/dead assays (Fig. 3(B)). The live/dead assay results show more than $85 \%$ of the cells were alive (appearing as green) at Day 1 and 3. Taken together, the results indicate that the fabricated fibers do not induce immediate toxicity and the presence of alginate with the tested concentrations does not reduce the cellular viability. In addition, to evaluate the cells morphology in the 3D structure of hybrid hydrogel, F-actin/DAPI staining was 
performed for the cells encapsulated in the hydrogel core at Day 1, 5, 7, and 17 (Fig. 3(C)). These findings demonstrate cell proliferation in a highly-organized pattern along with the fiber guideline. These results indicate the overall biocompatibility of the engineered hydrogel scaffold.

\subsection{Cellular alignment}

Cellular alignment is a critical consideration for in vitro neural tissue model systems. We observed that the hybrid hydrogel system significantly promotes cellular alignment along the fibers. Figure 4 shows the qualitative and quantitative results of the cellular orientation within the two-week periods after encapsulation. Fluorescent staining of neuroblastoma F-actin filaments indicates the alignment of the cells with respect to the fiber orientation (Fig. 4(A)). The orientation of F-actin filament was quantified and measured with respect to the direction of the channel. The results showed that after 7 days, actin filaments were oriented along the channel with the standard deviation of less than 10 degrees. The orientation was improved at Day 14 with the standard deviation of less than 6 degrees (Fig. 4(B)). The cell culture density within the 3D tubular structure was estimated at Day 7 and 14 per unit volume of the channel to compare the seeding density and those of the cells on the following days. The results showed that the seeding density was $15 \mathrm{million} / \mathrm{ml}$; whereas the density of cells after 7 and 14 days of culture was increased to 27 and $50 \mathrm{million} / \mathrm{ml}$, respectively (Fig. 4(C)).

\subsection{Numerical simulation}

Here, the shear stress occurring on the cells, and the cells confinement within the fiber's core during the extrusion are numerically simulated using $\mathrm{COMSOL}^{\circledR}$ Multiphysics. The geometry of two coaxial tubes with the inner diameters of $0.838 \mathrm{~mm}$ and $0.603 \mathrm{~mm}$, and the length of $12 \mathrm{~mm}$ was generated. The Navier-Stokes equation and a two-phase discrete phase model (DPM) were used to describe the fluid flow and the distribution of cells inside the tube, respectively. The simulations were conducted at different shell flow rates of 2,3 , and $4 \mathrm{ml} \mathrm{min}^{-1}$ for the constant core flow rate of $2 \mathrm{ml} \mathrm{min}^{-1}$. Figure 5 shows the cross-section view for the cells distribution and the shear stress on the cells surface at different shell flow rates $(2,3$, and $4 \mathrm{ml} / \mathrm{min})$ and distances from the nozzle head in the channel. Initially, the cells are distributed within the radius of $0<r<0.3 \mathrm{~mm}$. After passing through the channel, they concentrate within the region with $0<r<0.17 \mathrm{~mm}$. The pressure gradient arisen from the shell flow pushes the cells towards the center of the core stream. Figure 6 shows the shear stress distribution at different radius for three shell flow rates of 2,3 , and $4 \mathrm{ml} / \mathrm{min}$ along the channel (the channel side view). At first, a sudden jump in the shear stress profile is observed. However, after passing a critical length, the shear stress stays approximately constant.

\section{Discussion}


We speculate that the directed axonal outgrowth is obtained due to three phenomena: 1) the spatial confinement of the cells in the fibers and the stiffness of the core that applies mechanical stimulation to maintain the cells in a concentric distribution; 2) the alignment of polymeric sheets due to the flow induced shear stress during the extrusion process; and 3) the alignment of unmixed GelMA domains. GelMA and alginate do not mix homogeneously due to their dissimilar physical properties such as density, and thermal sensitive viscosity; since alginate hydrogel does not provide a cell friendly matrix, cells align toward the elongated GelMA networks (see Fig. 1). It has been shown that the size of hydrogel constructs play a key role in the morphological growth of the cells ${ }^{59,60}$. In smaller GelMA features, the cells can sense the boundaries of the GelMA microenvironment so that they align themselves within the boundaries. Here, due to the stiffness difference between the core and the shell, cells can align with the fiber's axis.

As it was shown in Fig. 4 (C), the cellular alignment together with the cellular density was increased after 14 days. The high density of the cells can be attributed to the highly oriented actin filaments observed on Days 7 and 14, as the higher density of the cells causes the elongation of the cells in the channel direction.

Although the use of neuroblastoma cells showed an excellent cell alignment and directional neural growth, these types of cells are not the best model for neural stem cells. Considering this fact, more investigations in needed to adjust the properties of the current system, including geometrical and chemical properties of the fibers, so it can prove its performance to recapitulate the in vivo environment.

Regarding the numerical simulations, it was shown that the system goes under a sudden jump in the shear stress, which after passing a critical length, it stays approximately constant. This suggests that increasing the shell flow rate delays the stress stabilization (longer critical length). As both Figs. 5 and 6 represent, the lowest shear stress value on the cells surface happens in the center of the channel and it increases gradually toward the channel walls. Moreover, the rise in shell flow rate causes the cells to experience higher shear stress.

The simulation results explain the stress-induced elongation of the polymer sheets due to flow extrusion force. Crosslinking of the hybrid hydrogel prevents the reorganization of these elongated structures. Thus, we speculated that the encapsulated cells were following this organization. The shear stress is higher close to the channel walls and therefore, elongation of the GelMA domains is higher in those regions.

\section{Conclusion}

A cell-laden composite hydrogel fiber constructed with tunable mechanical properties was fabricated. A microfluidic system was used to fabricate core-shell fibers with a cell-laden core and shell from a biocompatible cell-laden hybrid hydrogel of GelMA/gelatin. Despite many other studies on the application of coaxial bioprinting, the focus of this study was on the quantification of the cellular alignment and tunability of the scaffold constructs specifically for neural cells. The hydrogel fibers showed cell 
attachment, proliferation, and excellent growth within the fiber's core axis. The fibers directed the axonal outgrowth and generated a highly aligned cell pattern.

\section{Methods}

\subsection{Materials}

Sodium alginate, calcium chloride $\left(\mathrm{CaCl}_{2}\right)$, and DAPI were purchased from Sigma Aldrich (St. Louis, MO, USA). 2-hydroxy-1-(4-(hydroxyethoxy) phenyl)-2-methyl-1- propanone (Irgacure 2959, CIBA Chemicals) was used as photoinitiator (PI). Dulbecco's modified Eagle medium (DMEM), fetal bovine serum (FBS), 0.05\% trypsin-EDTA (1X), Live/Dead Assay kit, PrestoBlue ${ }^{\circledR}$ Cell Viability Assay, and antibiotics (Penicillin/Streptomycin) were purchased from Invitrogen (Carlsbad, CA, USA). GelMA was prepared according to our established protocol ${ }^{61}$.

\subsection{GelMA/gelatin hybrid hydrogel preparation}

Hybrid hydrogel was prepared by mixing the same volume of $2 \% \mathrm{w} / \mathrm{v}$ gelatin (in HBSS), $3 \% \mathrm{w} / \mathrm{v}$ GelMA (in HBSS) and finally adding $1 \% \mathrm{w} / \mathrm{v} \mathrm{PI}$ (in HBSS). Subsequently the hybrid solution was mixed for $10-20$ seconds with the vortex mixer at a low speed.

\subsection{Fabrication of hydrogel fibers}

To fabricate the hydrogel fibers, a co-flow device was utilized by assembling two needles of different sizes. The needles were chosen in a way that the outer dimeter of one is less than the inner diameter of the other one, where there would be a space between the needles for liquid to flow through. Here we used needle sizes of $18 \mathrm{G}$ and $20 \mathrm{G}$ to fabricate the device. The head of the needles were trimmed and flattened using sandpaper. An inlet (focusing flow inlet) was made in the plastic chamber of the bigger needle. The needles were then assembled together and glued. A transparent chemical resistant clear PVC tubing was then connected to the outlet and placed in a $2 \%$ calcium chloride solution in water.

As it is illustrated in Fig. 1, hydrogel fibers were fabricated using inner phase of 3\% GelMA mixed with $2 \%$ gelatin (different concentrations were made), and the focusing flow phase of $2 \%$ sodium alginate. The two phases were injected into the inlets of the microfluidic device. The flow rates of the solutions were finely controlled by syringe pumps (PHD 2000, Harvard Apparatus) to create fibers with different sizes. The hydrogel fibers were collected in a Petri dish containing $2 \%$ calcium chloride in water to fully crosslink the hydrogel. The fibers were then exposed to UV light (OmniCure s200, USA) for 30 seconds to crosslink the GelMA hydrogel in the fibers. The UV intensity was set at $850 \mathrm{~mW}$, and the distance between the tip of the fiber optic and the Petri dish was set at $8 \mathrm{~cm}$.

\subsection{Cell culture}

Neuroblastoma (SH-SY5Y) (ATCC, USA) were cultured in a 1:1 mixture of Dulbecco's Modified Eagle Medium (DMEM, Sigma), and F12 medium supplemented with $10 \% \mathrm{v} / \mathrm{v}$ fetal bovine serum (FBS, Sigma) 
and $1 \% \mathrm{v} / \mathrm{v}$ penicillin-streptomycin (Gibco, USA). Cells were maintained at $37^{\circ} \mathrm{C}$ in a humidified $5 \% \mathrm{CO}_{2}$ atmosphere until $80-90 \%$ confluence was reached. Cell passages 6-8 were used during experiments.

SH-SY5Y cells encapsulated in pre-described hybrid hydrogel using the following steps; after detaching cells (by trypsin), they were resuspended in cell culture media with the density of $15 \mathrm{M} \mathrm{mL}^{-1}$. The cells solution was added to the GelMA and gelatin hydrogel solution. Subsequently, GelMA/glatin containing the cell solution was mixed with PI solutions.

\subsection{Viability assay (Live/Dead)}

To evaluate the in vitro biocompatibility of the obtained hydrogel fibers, SH-SY5Y encapsulated in the hybrid hydrogel fibers were cut into smaller segments $(1 \mathrm{~cm})$ and placed in a 12-well plate. The viability test was performed by incubating the cell-laden samples for 15 minutes at $37^{\circ} \mathrm{C}$ with a mixture of $2 \mu \mathrm{l}$ $\mathrm{ml}^{-1}$ ethidium homodimer-1 (EthD-1, red, dead cells, Invitrogen) and $0.5 \mu \mathrm{ml}^{-1}$ calcein AM (live cells, green, Invitrogen). Fluorescence Microscopy was performed using a Zeiss Observer D1 microscope of an X-Cite Series $120 \mathrm{Q}$ fluorescence source.

\subsection{Actin/DAPI staining}

Samples were fixed in 4\% paraformaldehyde (Electron Microscopy Sciences), washed in DPBS and subsequently stained with phalloidin (life Technologies, labeled with Alexa F 594) and DAPI as described in the manufacturer's manual. Microscopy was performed as described above.

\section{References}

1. Shajahan, A. et al. Carbohydrate-Neuroactive Hybrid Strategy for Metabolic Glycan Engineering of the Central Nervous System in Vivo. J. Am. Chem. Soc. 139, 693-700 (2017).

2. Fawcett, J. W. The Paper that Restarted Modern Central Nervous System Axon Regeneration Research. Trends in Neurosciences. 41, 239-242 (2018).

3. Yiu, G. \& He, Z. Glial inhibition of CNS axon regeneration. Nat Rev Neurosci. 7, 617-627 (2006).

4. Anderson, M. A. et al. Astrocyte scar formation aids central nervous system axon regeneration. Nature. 532, 195-200 (2016).

5. Feigin, V. L. et al. The global burden of neurological disorders: translating evidence into policy. Lancet Neurol. 19, 255-265 (2020).

6. Mauffrey, P. et al. Progenitors from the central nervous system drive neurogenesis in cancer. Nature. 569, 672-678 (2019).

7. Gao, Y., Yang, Z. \& Li, X. Regeneration strategies after the adult mammalian central nervous system injury-biomaterials. Regen Biomater. 3, 115-122 (2016).

8. Sekine, Y. et al. Functional Genome-wide Screen Identifies Pathways Restricting Central Nervous System Axonal Regeneration. Cell Reports. 23, 415-428 (2018).

9. Adams, K. L. \& Gallo, V. The diversity and disparity of the glial scar. Nat Neurosci. 21, 9-15 (2018). 
10. Yang, F. et al. Genetic engineering of human stem cells for enhanced angiogenesis using biodegradable polymeric nanoparticles. Proc Natl Acad Sci USA. 107, 3317-3322 (2010).

11. Mead, B. P. et al. Focused Ultrasound Preconditioning for Augmented Nanoparticle Penetration and Efficacy in the Central Nervous System. Small. 15, 1903460 (2019).

12. Dawson, T. M., Golde, T. E. \& Lagier-Tourenne, C. Animal models of neurodegenerative diseases. Nature Neuroscience. 21, 1370-1379 (2018).

13. Lindvall, O., Barker, R. A., Brüstle, O. \& Isacson, O. \& Svendsen, C. N. Clinical translation of stem cells in neurodegenerative disorders. Cell Stem Cell. 10, 151-155 (2012).

14. Lee, H. \& Cho, D. W. One-step fabrication of an organ-on-a-chip with spatial heterogeneity using a 3D bioprinting technology. Lab on a Chip. 16, 2618-2625 (2016).

15. Omidinia-Anarkoli, A. et al. An Injectable Hybrid Hydrogel with Oriented Short Fibers Induces Unidirectional Growth of Functional Nerve Cells. Small. 13, 1702207 (2017).

16. Che, L., Lei, Z., Wu, P. \& Song, D. A 3D Printable and Bioactive Hydrogel Scaffold to Treat Traumatic Brain Injury. Adv. Funct. Mater. 29, 1904450 (2019).

17. Zhao, Y., Demirci, U., Chen, Y. \& Chen, P. Multiscale brain research on a microfluidic chip. Lab on a Chip. https://doi.org/10.1039/c9lc01010f (2020).

18. Nevill, J. T. et al. Vacuum soft lithography to direct neuronal polarization. Soft Matter. 7, 343-347 (2011).

19. Stratakis, E., Ranella, A., Farsari, M. \& Fotakis, C. Laser-based micro/nanoengineering for biological applications. Progress in Quantum Electronics. 33, 127-163 (2009).

20. Fasolino, I., Guarino, V., Cirillo, V. \& Ambrosio, L. 5-Azacytidine-mediated hMSC behavior on electrospun scaffolds for skeletal muscle regeneration: BEHAVIOR OF 5-AZACYTIDINE-MEDIATED HMSC. J. Biomed. Mater. Res. 105, 2551-2561 (2017).

21. John, J. V. et al. Engineering Biomimetic Nanofiber Microspheres with Tailored Size, Predesigned Structure, and Desired Composition via Gas Bubble-Mediated Coaxial Electrospray. Small. 16, 1907393 (2020).

22. Kim, J. H. et al. Neural cell integration into 3D bioprinted skeletal muscle constructs accelerates restoration of muscle function. Nature Communications. 11, 1-12 (2020).

23. Wang, X. et al. Coaxial extrusion bioprinted shell-core hydrogel microfibers mimic glioma microenvironment and enhance the drug resistance of cancer cells. Colloids and Surfaces $B$ : Biointerfaces. 171, 291-299 (2018).

24. Huang, C. Y., Hu, K. H. \& Wei, Z. H. Comparison of cell behavior on pva/pva-gelatin electrospun nanofibers with random and aligned configuration. Sci. Rep. 6, 1-8 (2016).

25. Bowser, D. A. \& Moore, M. J. Biofabrication of neural microphysiological systems using magnetic spheroid bioprinting. Biofabrication. 12, 015002 (2019).

26. GhavamiNejad, A., Ashammakhi, N., Wu, X. Y. \& Khademhosseini, A. Crosslinking Strategies for 3D Bioprinting of Polymeric Hydrogels. Small. 2002931, https://doi.org/10.1002/smll.202002931 
(2020).

27. Chircov, C. \& Grumezescu, A. M. Three-dimensional bioprinting in drug delivery. Materials for Biomedical Engineering. 2, 19-40 (2019).

28. Levato, R. et al. From Shape to Function: The Next Step in Bioprinting.Advanced Materials1906423, (2020).

29. Aljohani, W., Ullah, M. W., Zhang, X. \& Yang, G. Bioprinting and its applications in tissue engineering and regenerative medicine. International Journal of Biological Macromolecules. 107, 261-275 (2018).

30. Burnstine-Townley, A., Eshel, Y. \& Amdursky, N. Conductive Scaffolds for Cardiac and Neuronal Tissue Engineering: Governing Factors and Mechanisms. Adv. Funct. Mater. 1901369, https://doi.org/10.1002/adfm.201901369 (2019).

31. Naseer, S. M. et al. Surface acoustic waves induced micropatterning of cells in gelatin methacryloyl (GelMA) hydrogels. Biofabrication. 9, 015020 (2017).

32. Miri, K. A. et al. Bioprinters for organs-on-chips. Biofabrication. 0-22 (2019). https://doi.org/10.1088/1758-5090/ab2798 Manuscript

33. Arrabito, G., Ferrara, V., Bonasera, A. \& Pignataro, B. Artificial Biosystems by Printing Biology. Small. 16,1907691 (2020).

34. Dai, X. et al. Coaxial 3D bioprinting of self-assembled multicellular heterogeneous tumor fibers. Sci Rep. 7, 1457 (2017).

35. Uwamori, H., Higuchi, T., Arai, K. \& Sudo, R. Integration of neurogenesis and angiogenesis models for constructing a neurovascular tissue. Sci. Rep. 7, 1-11 (2017).

36. Knowlton, S., Anand, S., Shah, T. \& Tasoglu, S. Bioprinting for Neural Tissue Engineering. Trends in Neurosciences. 41, 31-46 (2018).

37. Lozano, R. et al. 3D printing of layered brain-like structures using peptide modified gellan gum substrates. Biomaterials. 67, 264-273 (2015).

38. Papadimitriou, L., Manganas, P., Ranella, A. \& Stratakis, E. Biofabrication for neural tissue engineering applications. Materials Today Bio. 100043, https://doi.org/10.1016/j.mtbio.2020.100043 (2020).

39. McMurtrey, R. J. Patterned and functionalized nanofiber scaffolds in three-dimensional hydrogel constructs enhance neurite outgrowth and directional control.Journal of Neural Engineering11, (2014).

40. Heinrich, M. A. et al. 3D Bioprinting: from Benches to Translational Applications. Small. 1805510, https://doi.org/10.1002/smll.201805510 (2019).

41. Liu, D., Shi, Q., Dai, S. \& Huang, J. The Design of 3D-Interface Architecture in an Ultralow-Power, Electrospun Single-Fiber Synaptic Transistor for Neuromorphic Computing. Small. 16, 1907472 (2020). 
42. Innala, M. et al. 3D Culturing and differentiation of SH-SY5Y neuroblastoma cells on bacterial nanocellulose scaffolds. Artificial Cells, Nanomedicine and Biotechnology. 42, 302-308 (2014).

43. Kuzmenko, V., Karabulut, E., Pernevik, E., Enoksson, P. \& Gatenholm, P. Tailor-made conductive inks from cellulose nanofibrils for 3D printing of neural guidelines. Carbohydr. Polym. 189, 22-30 (2018).

44. Li, Y. et al. Engineering cell alignment in vitro. Biotechnol. Adv. 32, 347-365 (2014).

45. Arrigoni, C. et al. Engineering complex muscle-tissue interfaces through microfabrication. Biofabrication11, (2019).

46. Li, X. et al. A comparative study of the behavior of neural progenitor cells in extrusion-based in vitro hydrogel models. Biomedical Materials (Bristol). 14, 65001 (2019).

47. Wang, X. et al. Coaxial extrusion bioprinted shell-core hydrogel microfibers mimic glioma microenvironment and enhance the drug resistance of cancer cells. Colloids and Surfaces B: Biointerfaces. 171, 291-299 (2018).

48. Li, X. et al. A coaxially extruded heterogeneous core-shell fiber with Schwann cells and neural stem cells. Regenerative Biomaterials. 131-139 https://doi.org/10.1093/rb/rbz037 (2019).

49. Brook, G. A. et al. Spontaneous longitudinally orientated axonal regeneration is associated with the Schwann cell framework within the lesion site following spinal cord compression injury of the rat. Journal of Neuroscience Research. 53, 51-65 (1998).

50. Onoe, H. et al. Metre-long cell-laden microfibres exhibit tissue morphologies and functions. Nature Mater. 12, 584-590 (2013).

51. Salaris et al. 3D Bioprinted Human Cortical Neural Constructs Derived from Induced Pluripotent Stem Cells. JCM. 8, 1595 (2019).

52. Kjar, A., McFarland, B., Mecham, K., Harward, N. \& Huang, Y. Engineering of tissue constructs using coaxial bioprinting. Bioactive Materials. 6, 460-471 (2021).

53. Sarker, M., Naghieh, S., McInnes, A. D., Schreyer, D. J. \& Chen, X. Strategic Design and Fabrication of Nerve Guidance Conduits for Peripheral Nerve Regeneration. Biotechnol. J. 13, 1700635 (2018).

54. Yue, K. et al. Synthesis, properties, and biomedical applications of gelatin methacryloyl (GelMA) hydrogels. Biomaterials. 73, 254-271 (2015).

55. Fallahi, A., Khademhosseini, A. \& Tamayol, A. Textile Processes for Engineering Tissues with Biomimetic Architectures and Properties. Trends in Biotechnology. 34, 683-685 (2016).

56. Mostafalu, P. et al. Smart flexible wound dressing with wireless drug delivery. in 2015 IEEE Biomedical Circuits and Systems Conference (BioCAS) 1-4 (IEEE, 2015). doi:10.1109/BioCAS.2015.7348391.

57. Shin, S. J. et al. "On the Fly" Continuous Generation of Alginate Fibers Using a Microfluidic Device. Langmuir. 23, 9104-9108 (2007).

58. Leipzig, N. D. \& Shoichet, M. S. The effect of substrate stiffness on adult neural stem cell behavior. Biomaterials. 30, 6867-6878 (2009). 
59. Li, H. et al. Bioengineered three-dimensional scaffolds to elucidate the effects of material biodegradability on cell behavior using POSS-PEG hybrid hydrogels. Polymer Degradation and Stability. 164, 118-126 (2019).

60. Nejad, R. H. et al. Laterally Confined Microfluidic Patterning of Cells for Engineering Spatially Defined Vascularization. Small. 12, 5132-5139 (2016).

61. Nichol, J. W. et al. Cell-laden microengineered gelatin methacrylate hydrogels. Biomaterials. 31, 5536-5544 (2010).

\section{Figures}

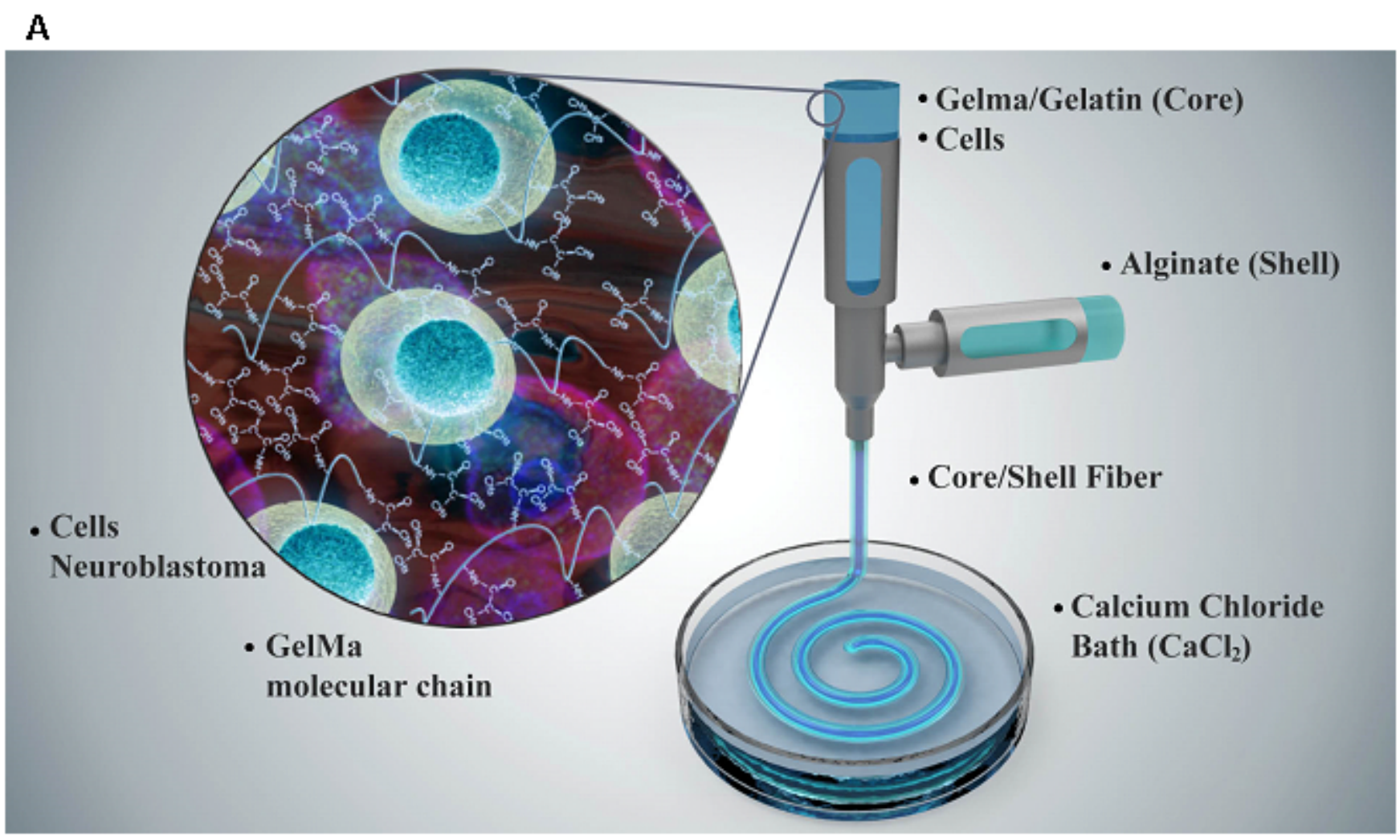

B

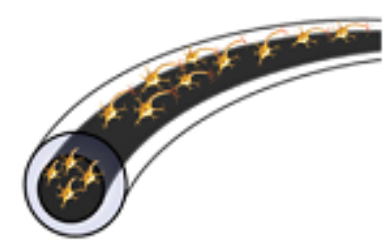

Core: cell laden hydrogel Shell: hydrogel
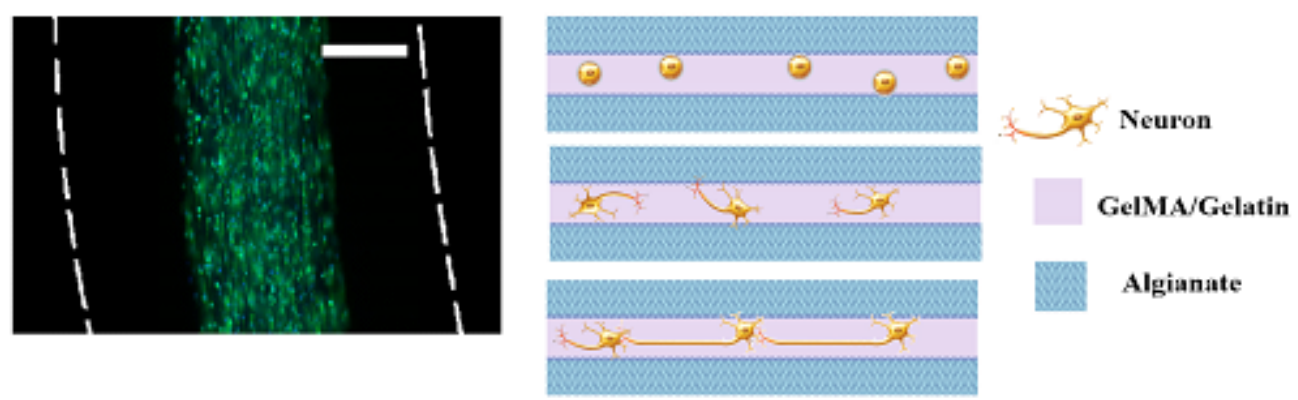

\section{Figure 1}

The schematic view of the high throughput fabrication process of the core-shell hydrogel fiber and the resulted construct. (A) The setup is consisted of a microfluidic device with the ability to control the flow rate of the core and shell solution, separately. The outlet is injected into the $\mathrm{CaCl} 2$ bath where alginate is 
crosslinked to form the fiber's matrix. Crosslinked alginate traps the hydrogel in the core (GelMA/gelatin) and forms a template network in the shell. (B) The samples were exposed to UV-irradiation for crosslinking GelMA. Representative micrographs showing produced fiber. Scale bar is $500 \mu \mathrm{m}$. The schematic of neural fiber generation; alginate acts as a template preventing neurons from diagonal expansion and confines them in the GelMA/gelatin core resulting in a directed neurite outgrowth.
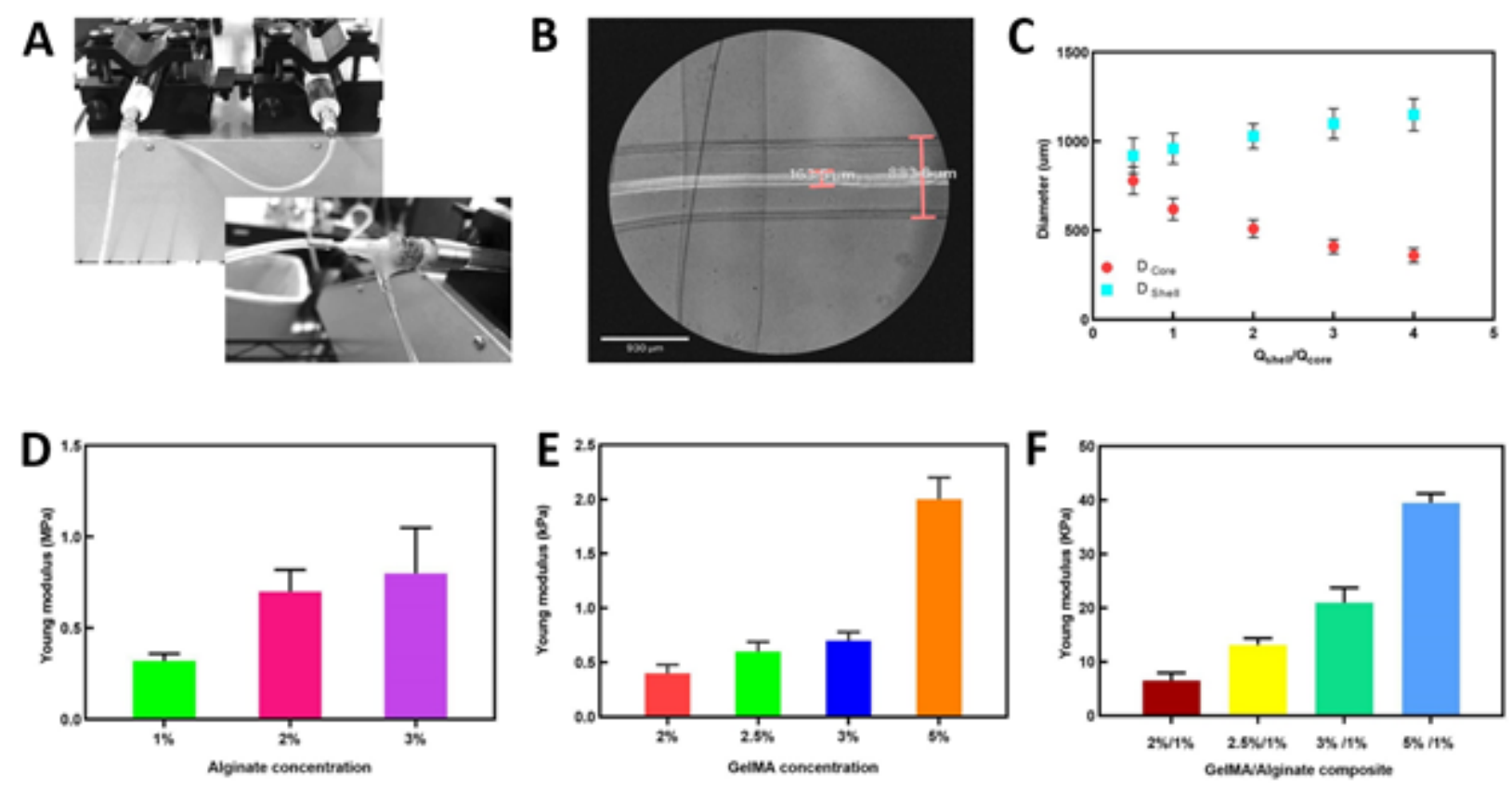

Figure 2

Characterization of hydrogel fibers. (A) The fabrication setup of core-shell hydrogel fibers. (B) The geometrical features of core-shell hydrogel fibers as compared to sole hydrogel fiber. (C) The diameter can be easily controlled by adjusting the core and sheath flow rates. (D) The Young's modulus for microfibers with different alginate concentration. (E) The Young's modulus of GelMA/gelatin hydrogel for four levels of GelMA concentration; the gelatin concentration was kept constant as $1 \%$ for all. (F) The Young's modulus for different concentrations of GelMA combined with $1 \%$ alginate. For all three mechanical tests: P-Value $<0.05$. 

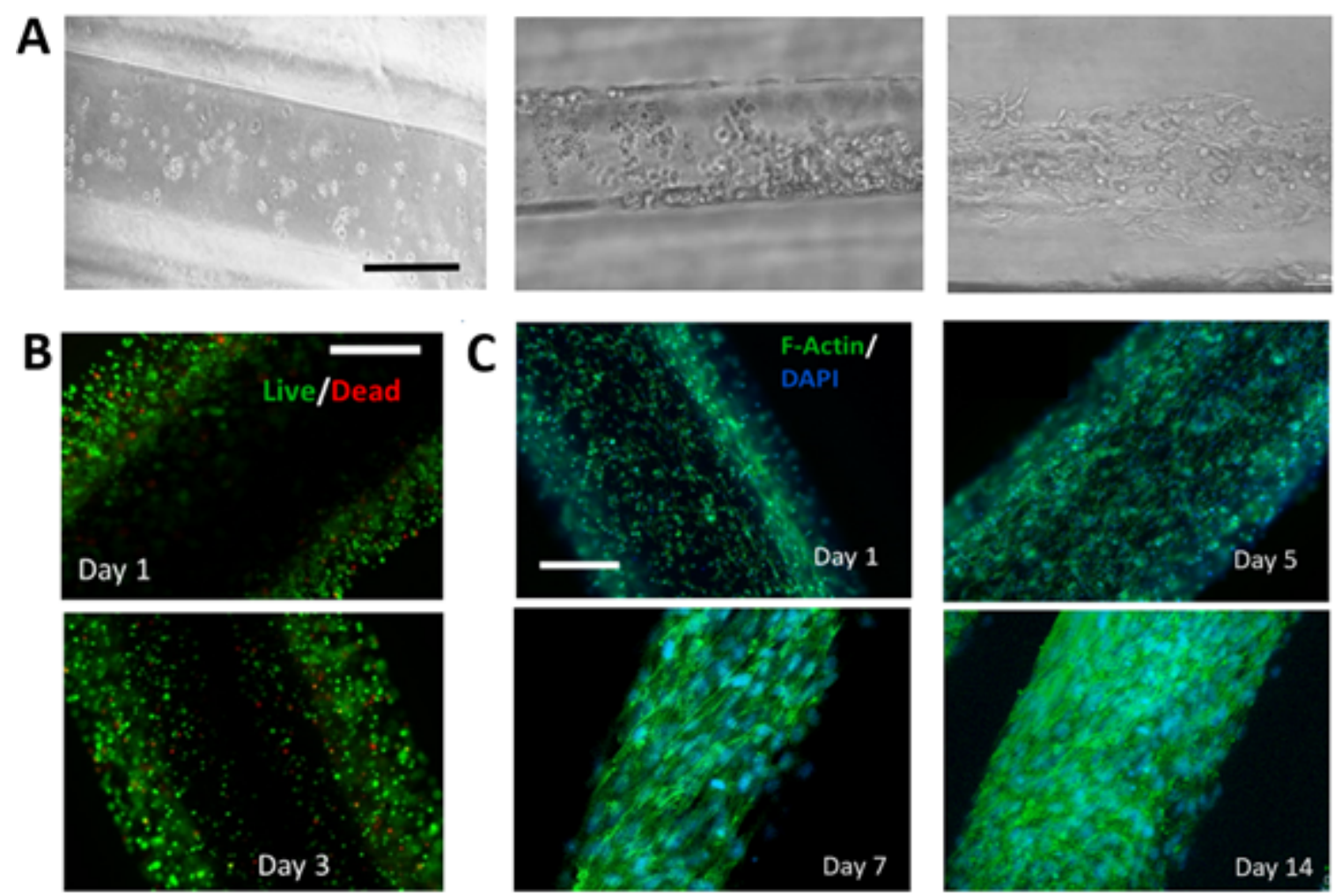

Figure 3

Viability and proliferation of neuroblastoma encapsulated in the fibers. (A) Phase-contrast images of neuroblastoma encapsulated in hydrogel fiber at day 0 , day 1 , and day 5 , from left to right respectively (scale bar $100 \mu \mathrm{m}$ ). (B) Live/dead staining of neuroblastoma cells at day 1, and day 3 (scale bar 100 $\mu \mathrm{m}$ ). (C) F-actin/DAPI staining at different days of culture, day 1, day 5, day7, and day 14 (scale bar 50 $\mu \mathrm{m})$.


\section{Figure 4}

The qualitative and quantitative analysis of cellular orientation in different days of the culture for the encapsulated cells in the fiber. (A) F-actin/DAPI staining after 7 and 14 days, respectively from left to right. (B) Deviation of neuroblastoma from the channel direction for Day 7 and 14. (C) Cell seeding density and culture density after Day 7 and 14 (scale bar $25 \mu \mathrm{m}$ ). 


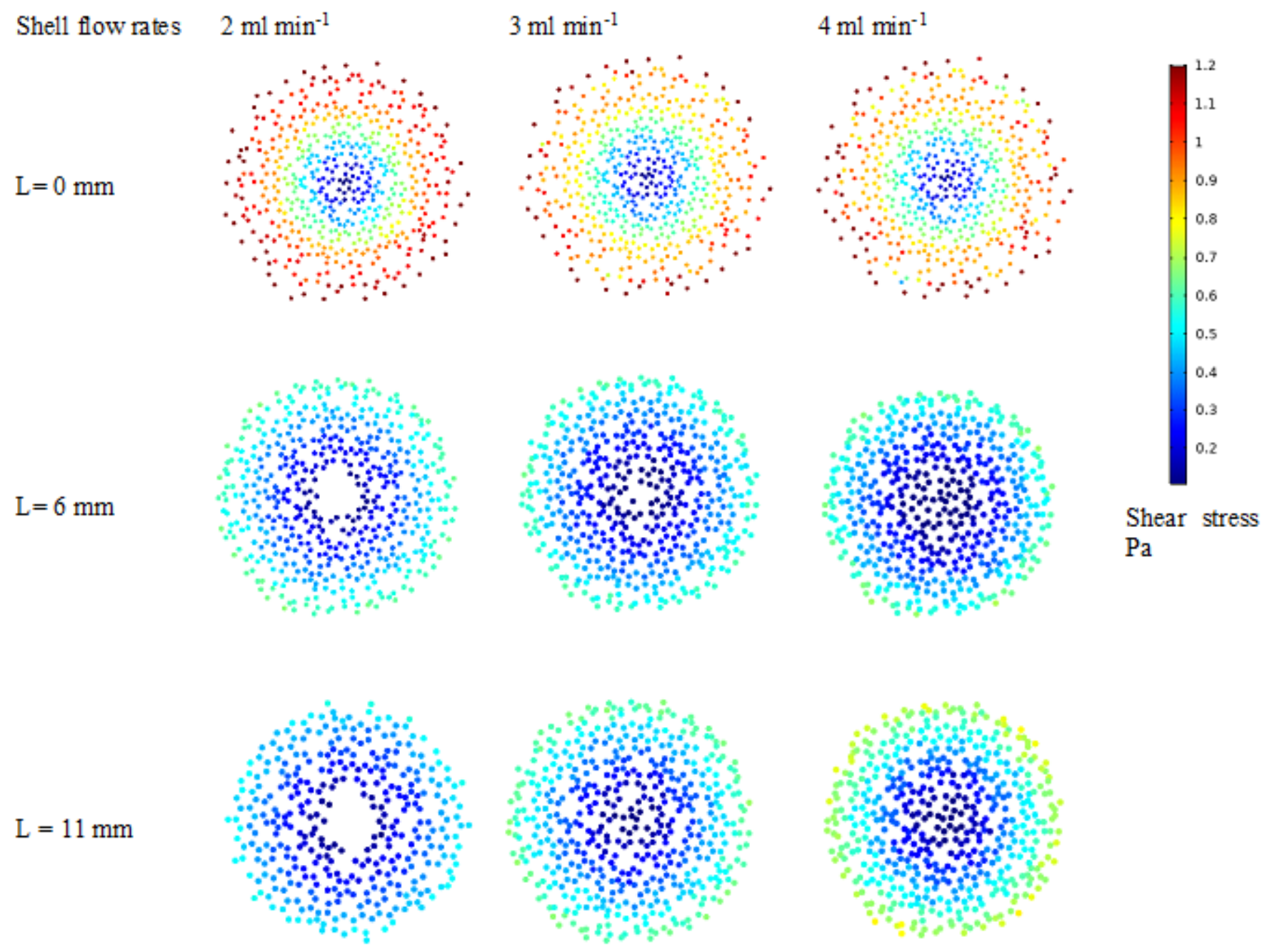

Figure 5

Cross section view of the shear stress distribution on the cells and cell dispersion at different distances from the nozzle head $(0,6$ and $11 \mathrm{~mm})$. 
A



B

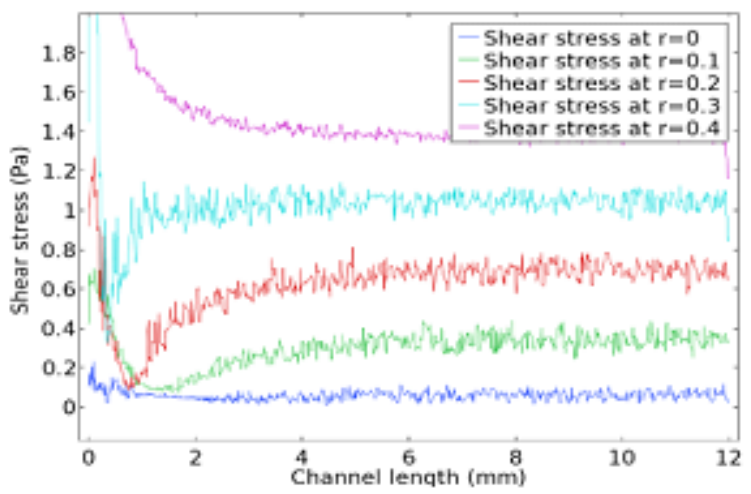



Figure 6

Shear stress at different radius (across the cross section of the coaxial nozzle) and shell flow rates along the tube. Shell flow rate is: (A) $2 \mathrm{ml} \mathrm{min-1}$ (B) $3 \mathrm{ml} \mathrm{min-1}$ (C) $4 \mathrm{ml}$ min-1. At $r=0$ (center of the core flow) shear stress is minimum, while at $r=0.4$ (core/shell interface) shear stress reaches the maximum value. 